\title{
The visual and measured effects of high-velocity motion and a effective reconstruction method of physical field under the limited velocity of light
}

Xiao Yuxiang ( $\sim$ xiaoyuxiang1999@163.com )

Northeast University at Qinhuangdao https://orcid.org/0000-0002-0972-166X

Peng Xiao

Tymphany co., Ltd

Article

Keywords:

Posted Date: January 20th, 2022

DOI: https://doi.org/10.21203/rs.3.rs-1248667/v1

License: (c) (i) This work is licensed under a Creative Commons Attribution 4.0 International License.

Read Full License 


\section{The visual and measured effects of high-velocity motion and a effective reconstruction method} of physical field under the limited velocity of light

(1)

\section{Abstract}

In two frame of reference with relative motion, observing each other's clocks and rulers is an important method to judge whether they have changes in time rate and length. The light reflection device (including light source, mirror opposite the light source and observer) is a suitable clock and ruler.

However, people have been living in low-velocity motions world, so it is easier to cause some confusions. For example, because the velocity of light is limited, in a high-velocity train it is impossible for a directed single or a series of photons to reach the mirror opposite the device and the mirror is not in the expected rest position. It can only succeed when it emits the spherical light waves. However, at this time, both high-velocity and rest observers can detect the same light path and time.

On the other side, if the device is set on the ground, both high-velocity and rest observers also detect the same light path and time which are both decreased. Therefore, the time rate is faster and the length is longer than that on the train.

The article uses the physical field reconstruction to study the physical process of high-velocity motion, and points out that the geometric center of the light emitting can be used as an absolute rest frame of reference to analysis.

The proposed new concept of space-time can deal with the Twin Paradox, and explain the highvelocity physical phenomena observed.

\section{Keywords}

Velocity of light; Ether; Proper time; Synchronous clocks; Special Relativity; Twin Paradox; Time dilation; Length contraction; Photon; Spherical light waves. 
1

2

3

4

\section{Article}

\section{The premise}

This article discusses all are based on the current calculations and observed results as the premise:

1.1 Light is an electromagnetic wave, without the ether as the transmission medium, it propagates in a vacuum as a field, and its velocity in vacuum measured in any Inertial system (normally is rest) along each direction is a constant value, this result also calculated from Maxwell's set of equations. Recorded as constant $C=3 * 10^{8} \mathrm{~m} / \mathrm{s}$.

1.2 The velocity of rays emitted by high-velocity mesons are still the $C$, which means $C$ is a limited velocity (both particles and information transmission) in the world, and the light is always propagated in empty space with a definite velocity $\mathrm{C}$ which is independent of the state of motion of the emitting body, said by Albert Einstein.

Note: The visual effects we discussed are excluding perspective.

\section{The current opinions}

As in [1], assume that there is the light source and a mirror which is opposite the light source on a high-velocity train running at a constant velocity $\mathrm{V}$, as shown in Figure $1 \mathrm{a} \& 1 \mathrm{~b}$. The light source emits light (the light is shown in red in the figure) as event 1 , and the light reflects to the light source as event 2 .

Therefore, the observer Mr. Train (Figure 1a) on the high-velocity train will only use one clock to record the time difference between the two events. Since both events seems occur locally, they are recorded as $\Delta t_{0}$ called the proper time. The distance traveled by the light is $2^{*} \mathrm{D}$.

The observer on the ground, Mr. Ground (as shown in Figure 1b) observes that Event 1 and Event 2 occur in different places. Because the light has taken a longer path $(2 * \mathrm{~L})$ in this observation, and since the velocity of light is constant, therefore, according to the calculation in the literature, the measured $\Delta \mathrm{t}$ is:

$$
\Delta t=\gamma \Delta t_{0}
$$

$$
\text { and } \quad \gamma=\frac{1}{\sqrt{1-\left(\frac{V}{C}\right)^{2}}}
$$


1

2

3

4

5

6

8

10

11

12

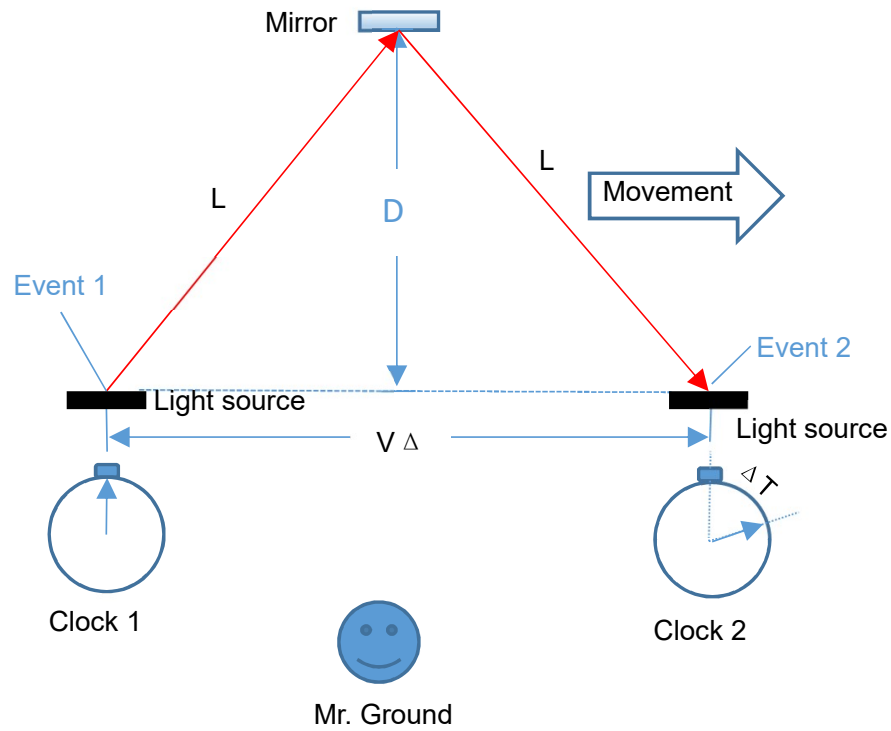

Figure 1b. Result of the observer on the ground.

Mr. Train

Figure 1a. Result of the observer on the train.

As long as $V$ is not 0 , the measured $\Delta t$ is always larger than $\Delta t_{0}$, which is called the time dilation of the Special Relativity, the length contraction can be derived from the time dilation.

However, it is generally believed that the time rate of people on the ground observed by people on the train will also be slowed down. This is caused by the relativity of motion, and it is also the root cause of the Twin Paradox.

\section{The visual and measured effects of high-velocity motion}

However, people have been living in low-velocity motions, and they often judge the physical phenomena of high-velocity motion intuitively, which is not rigorous enough.

For better understanding for the visual effects of the device in high-velocity train, we can set the distance $D$ between the mirror and the light source to 1 light-year. Due to the diffusion state after the light is emitted is not affected by the motion state of the light source, the directed single photon from light source needs to reach the original position of the mirror after 1 year, but in fact, the mirror has long been far away, so there is no reflected light at all, and the experiment of the device is unsuccessful whether is for observers on the train or ground, as shown in Figure 2a. 


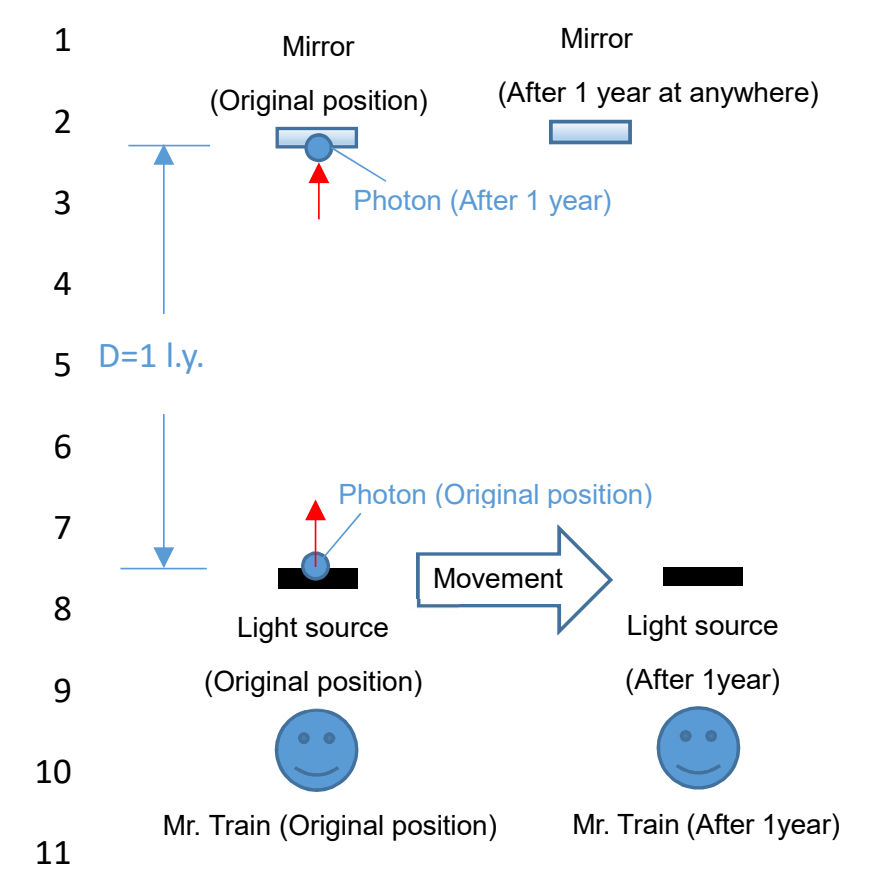

Figure 2a. The directed photon cannot reach the mirror after 1 year. dashed line in Figure $2 b$ will need to be explained later. of the light path observed on the ground is $2^{*} \mathrm{~L}$. train is also $2^{*} \mathrm{~L}$ instead of $2^{*} \mathrm{D}$ in the traditional opinion.

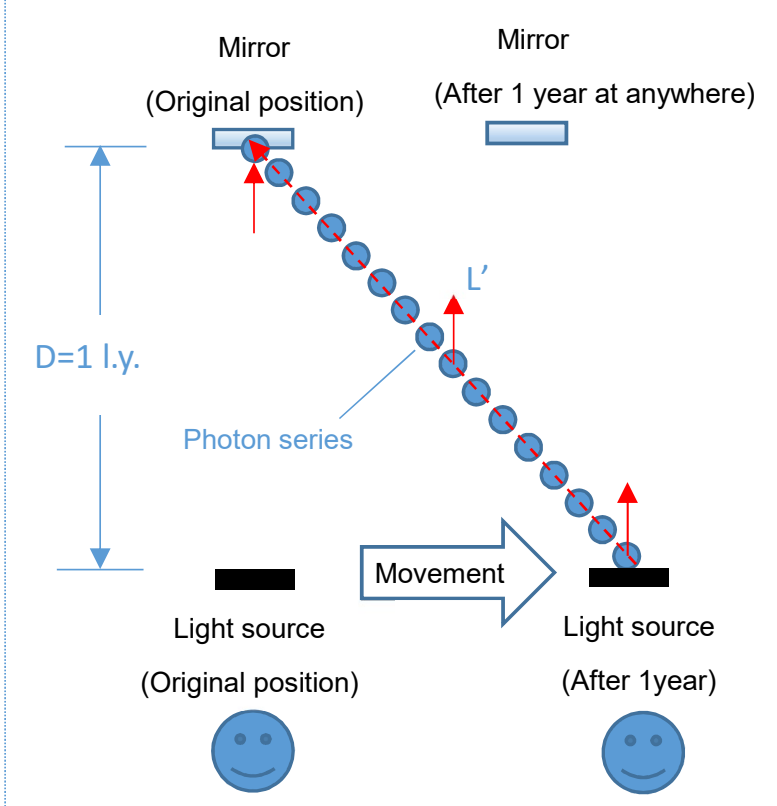

Mr. Train (Original position)
Figure 2b. The visual trace of a series of photons.

If the light source is a directed continuous photon series, the oblique trace L' represented by the

This is one of the common misunderstandings. Not only that, we will also propose later in the analysis that for the observer in motion, the mirror is not directly in front of the light source.

The basis for the real success of the experiment is that the light source emits spherical light waves, as shown in Figure 3 below the spherical light waves (indicated by the blue dashed line) always reach the mirror then reflect to the light source. For convenience, the simplified light path can be drawn as straight line, shown in Figure 3 , the same as in the previous Figure $1 \mathrm{~b}$, the length

The smart experts can recognize immediately the length of the light path observed on the

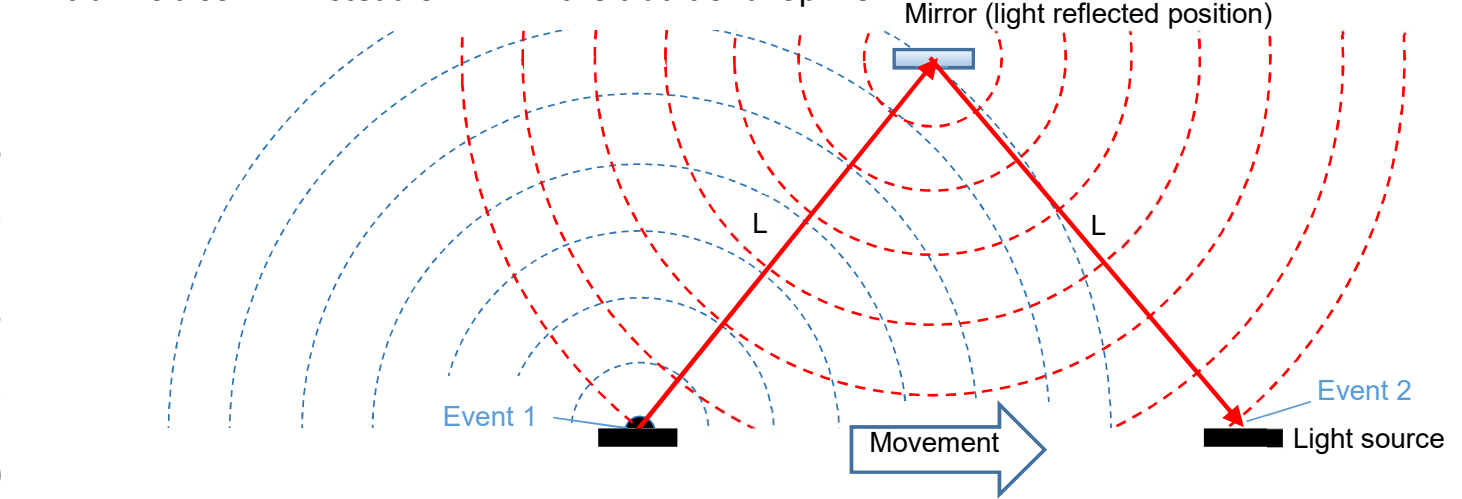

Figure 3. The spherical light wave reflection observed by Mr. Ground 
But before proceeding to the next detailed analysis, we would like use an example to redefine

2 the rules because we found a lot of confusion.

\section{4. The use of synchronized clock for physics field reconstruction}

$4 \quad 4.1$ The use of synchronized clock

5 We suppose there is a planet B which is 1 light-year ( $L=1$ I.y.) away from observer A (the planet 6 is relatively static with the observer), and a rocket with a velocity of $0.8 \mathrm{C}$ is launched to A, shown in Figure 4a, and it takes 1 year for the wavefront of launch event to reach the observer. The rocket

8 takes 1.25 years, shown in Figure 4b.We use the following Table1 to list the sequence of events observed by A:

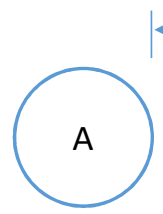

$$
1
$$$$
D=1 \text { l.y. }
$$

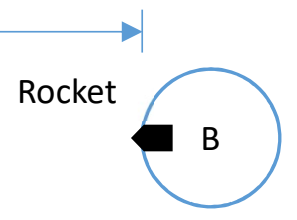

Figure 4a. $B$ launch the rocket to $A$

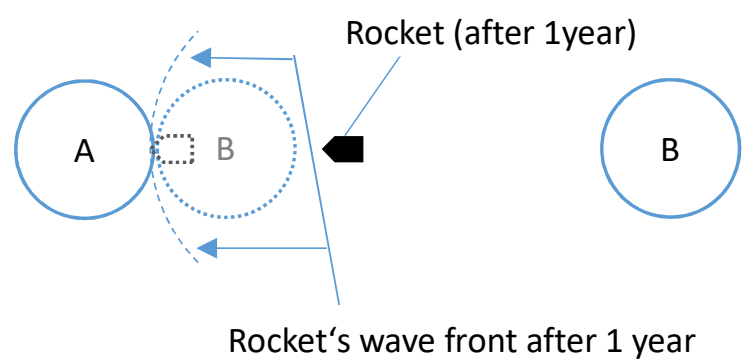

Figure 4b. The wave front and exact position of the rocket after 1 year

Table 1

\begin{tabular}{|c|c|c|}
\hline Event & Location & Clock \& Time \\
\hline A rocket launches to A & Planet B & At a certain moment, such as 2023.01.01, am \\
& & $8: 00$ \\
\hline The rocket arrives at A & A & 0.25 years after above moment \\
\hline
\end{tabular}

If the observer just do 2 times effective observations and records (means that he does not know the exact distance between A to B), in fact he cannot calculate the velocity, which needs to add an event 0 to measure the distance.

Then we can calculate the velocity of the rocket as:

$$
V^{\prime}=L / t=1 \text { l.y. } / 0.25 \text { year }=4 C
$$

This severely violates the laws of that the velocity of light is limited. It means the vision events cannot use for physics analysis. That's why we should introduce the concept of the synchronized clock. 

the actual time of the event can be known, then the sequence of events recorded by observer $A$ according to the synchronized clock is:

4 Table 2

\begin{tabular}{|c|c|c|}
\hline \multicolumn{1}{c|}{ Event } & Location & Clock \& Time \\
\hline $\begin{array}{c}\text { A sends a radio waves ranging to B, and } \\
\text { the estimated distance is 1 light-year }\end{array}$ & A & For the synchronized clock \\
\hline A rocket launches to A & Planet B & At a certain moment, such as \\
\hline The rocket arrives at A & 2023.01.01, am 8:00 \\
\hline
\end{tabular}

5 Calculate the rocket velocity:

$$
V=L / t=1 \text { I.y. } / 1.25 \text { years }=0.8 \mathrm{C}
$$

7 So we must use the synchronized clock for physics field reconstruction.

\section{$8 \quad 4.2$ Key frame method}

9 In order to facilitate analysis, we can use multiple key frames combined with the synchronized clocks, following by some suggestions.

11 A. For multiple key frames such as T0, T1,.., Tn, put the objects on each key positions, and 12 describe the movement directions, the T1-Tn must use the result of synchronous conversion.

B. Use the isochrone to connect multiple objects (If need).

C. Draw the propagation direction of the spherical light wave or position of the photon, and the wave front path transmitted to the observer (If need).

D. Stack multiple key frames by the alignment rest piont for physics field reconstruction .Similarly, in the visual fields reconstruction, we need align based on the observer. Figure 5. 


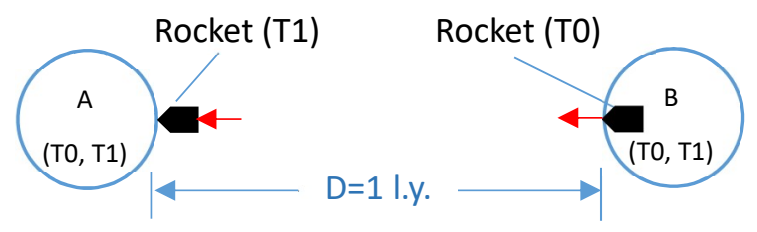

$\mathrm{T} 1-\mathrm{T} 0=1.25$ years

Figure 5. Stacked key frames for physics field reconstruction

In a word, for the research and description of all problems, it should be stated whether the description is a visual field or a physics field reconstruction in advance.

\section{The physics field reconstruction of the observer on train}

Back to the discussion of the visual phenomena seen by the observer on the train under the spherical light wave. We assume that the velocity of the train is $v=0.8 \mathrm{C}$. Since $\mathrm{D}$ is 1 light-year, although the physical position of the mirror can be considered to be directly opposite to the light source at the rest, since the velocity of light is limited, the observer can only receive the light waves from the mirror more than 1 year ago (similar to the sun we can only observe 8 minutes ago), that is the fact that the mirror is not directly in front of the light source (both visual and physical measurements), but it is located directly behind the light source a distance position(The direction opposite to the direction of train movement).

Then we start the physics field reconstruction as Figure 6, assume that there is smoke in the space to facilitate observation of the light path.

We divide the spatial distribution of the observer and the light diffusion into 11 key frames T0T10, since the diffusion state of light is not affected by the movement of the light source, the wave front of the part of the light participating in the reflection can be drawn sequentially and fixed in the figure, and then draw the wave front and the observer position of each key frame. The positions are connected as the isochrone (the blue dashed line in the figure), and then the path of wave front position of the light reflected to the observer is drawn (the black center line in the figure), the light reflection is exactly located in the even-number frames on the position of Mr. Train.

In other words, what Figure 6 wants to express is that when he move to the even-numbered frame T2-T10 position, he can observe that the light wave front position is exactly at the position of T1-T5. 


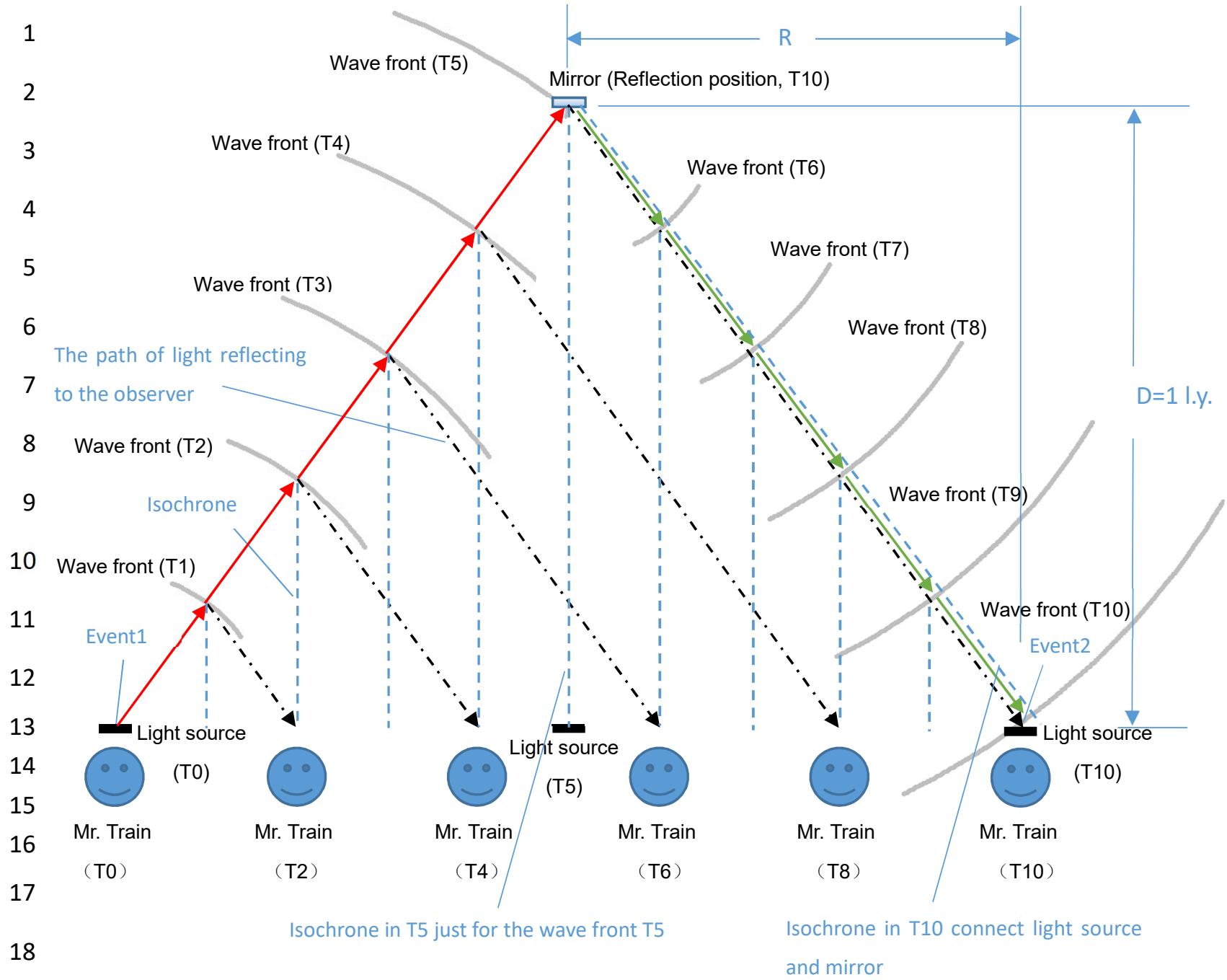

19 Figure 6. Atter physics tield reconstruction, in the stacked 11 key frames of the 20 observer during the motion.

When the observer is at T10, the wave front $\mathrm{T} 5$ of the event "light reaches the mirror" is transmitted to the observer, and the light reflected from the mirror also reaches the observer at the same time. This is an interesting phenomenon in which light is used for testing and at the same time only light can be used to observe objects. It is very important to realize this, because it is destined that the mirrors observed by regardless the train observer or the ground observer at the moment of event 2 are all located in the same location. It is not difficult to calculate $\mathrm{R}$ and there is still a distance offset even when $D$ is very small.

$$
R=\frac{\gamma^{*} D^{*} V}{C} \text { Where } \mathrm{D} \text { is rest distance of the mirror }
$$

The physics field reconstruction (Figure 6) can be used to analysis. Obviously, the observers on the train or on the ground also think that the distance traveled by the reflected light is $2 * \mathrm{~L}$. 


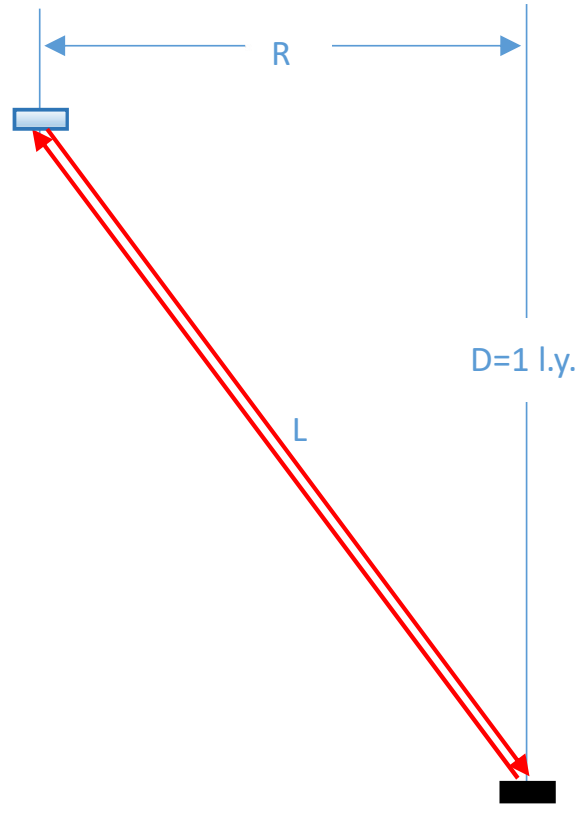

Mr. Train

(T0-T10)

Figure 7. The stacked visions of the observer on the train

In order to get the observer's visual effect, we can re-align the key frames of Figure 6 again according to his position, then see the mirror he observed is not directly in front of him, but is located directly behind a distance position. If the light source emits a bunch of photons, its path in space will follow the red line in Figure 7 until it reaches the mirror at frame T10 as shown in Figure 6, and for him, the reflection angle of light is not as understood which is in the rest world.

The light path in Figures 6 and 7 is continuous in space, and the lengthening effect caused by it is a high-velocity motion effect which is the result of actual measurement.

This oblique laser path is not the same as the red dashed line in Figure $2 \mathrm{~b}$. In Figure $2 \mathrm{~b}$, although the length of the dashed line $L^{\prime}$ is larger than $D$ (1 light-year), the first photon still just reaches the original position of the mirror from the original position of the light source, and the distance of photon is 1 light-year.

For the observer on the train, he has no way to detect whether time is slowing down. For him, time passes normally, but some physical phenomena are different from those at rest.

In order to compare the movement clock changes of the two frame of references, two observers are required to observe each other's clocks for comparison.

For example, in the above behavior, assume the train velocity is $0.6 \mathrm{C}$, then $\gamma=1.25$. Table 3 reflects the results obtained by ground observers after observing the ground device and the train device: 


\begin{tabular}{|c|c|c|c|}
\hline Observer & $\begin{array}{c}\text { Device on } \\
\text { the ground }\end{array}$ & $\begin{array}{c}\text { Device on } \\
\text { the train }\end{array}$ & \multicolumn{1}{c|}{ Conclusion } \\
\hline Mr. Ground & 2 years & 1.6 years & $\begin{array}{l}\text { Mr. Ground thinks that the time rate on the ground } \\
\text { is } 1.25 \text { times that on the train }\end{array}$ \\
\hline
\end{tabular}

But as we mentioned above, we also need to carefully check the observer on the train observing

3 the clock on the ground.

$4 \quad$ For the vision of observer on the train, the ground device moves away from him at a speed V (in

5 the direction of the dashed arrow in Figure 8). For the convenience of analysis, 5 key frames (T0-

6 T4) with Mr. Train as the frame of reference are stacked in Figure 8. And the light source is set to a

7 single photon, which has already succeed in this case.

8 We can see the photon forms an visual oblique trace in space with a red dotted line indicating

9 in Figure 2, but in fact it is not such a light path whether the light source emits a single or continuous photon, spherical wave (it is also a major misunderstanding of the traditional interpretation of the Special Relativity), and because the diffusion state after the light is emitted is not affected by the motion state of the observer or the light source, the true path of the photon is as shown in the red arrow in Figure 8, it is directly emitted to the rest position of the mirror and reflected back, so the path that the light travels is still $2^{*} D$, which is exactly the same as the result of the ground observer.

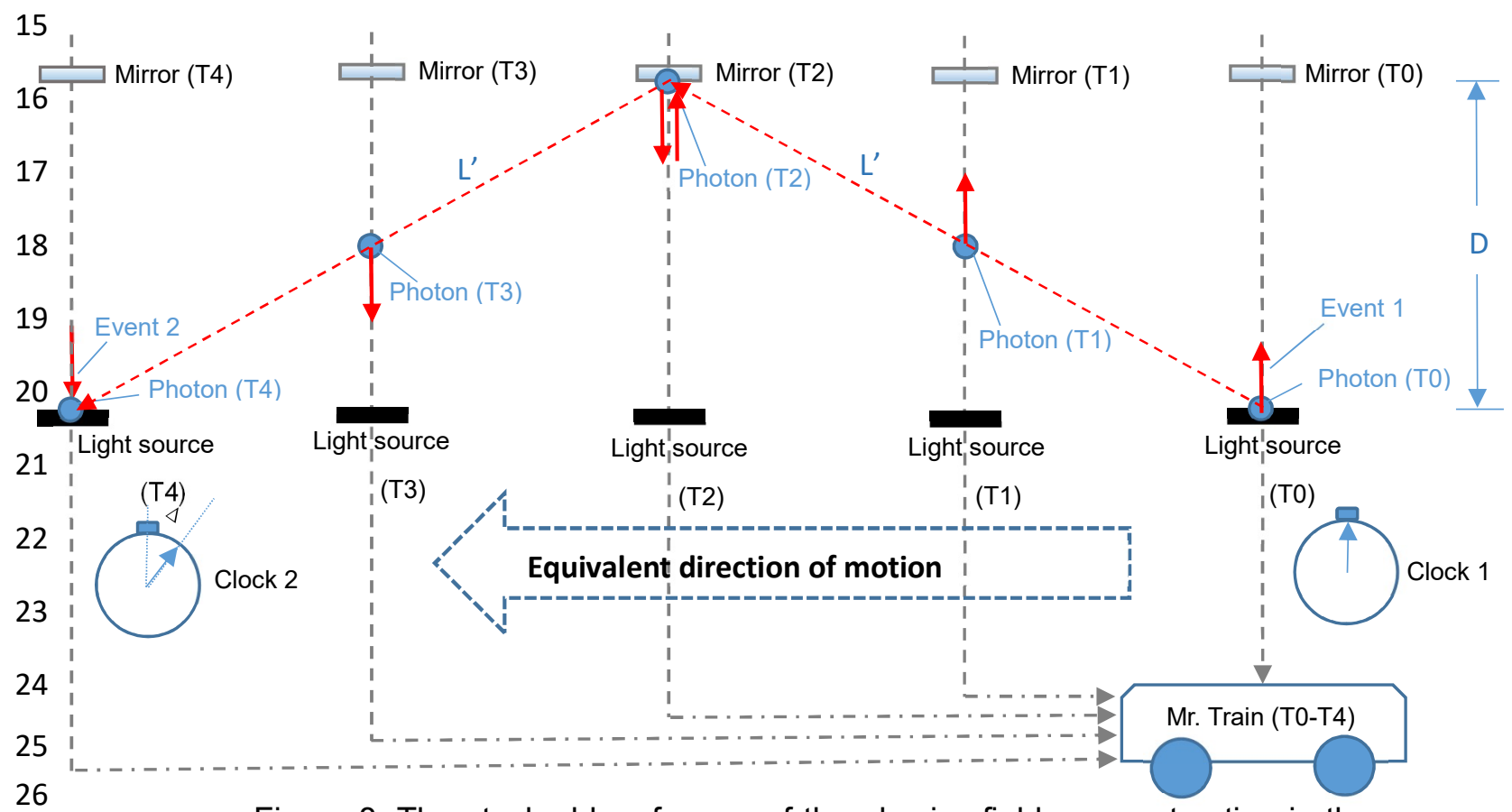

Figure 8. The stacked key frames of the physics fields reconstruction in the frame of reference (Mr. Train) 
Therefore, the train observer's report on the device on the train and ground is shown in Table 4:

Table 4

\begin{tabular}{|c|c|c|l|}
\hline Observer & $\begin{array}{c}\text { Device on } \\
\text { the train }\end{array}$ & $\begin{array}{c}\text { Device on the } \\
\text { ground }\end{array}$ & \multicolumn{1}{c|}{ Conclusion } \\
\hline Mr. Train & 2 years & 2.5 years & $\begin{array}{l}\text { Mr. Train thinks that the time rate on the ground } \\
\text { is } 1.25 \text { times that on the train }\end{array}$ \\
\hline
\end{tabular}

There is no contradiction between the observations and conclusions in Table 3 above.

In fact, for a non-moving observer, considering that he continuously uses the synchronized clocks

to follow the test continuously, it is actually equivalent to playing the role of a motion observer by constantly following the motion. In other words, the synchronized clock only compensates for the light propagation time from the non-moving observer to the location of the event, and does not affect any other physical states. That non-moving and moving result from observed or measured is exactly same.

\section{Time dilation and length contraction}

\subsection{Time dilation}

Because of this, the name or concept of proper time must be discarded. It can be represented by the rest time (TO), while the time of the motion frame of reference is represented by Td, And the relationship between the two is:

$$
T d=T O / Y
$$

\subsection{Length change}

Obviously, the clock device can also be used as a standard ruler carried. (In fact, now we define the length of light traveled in $1 / \mathrm{C}$ seconds as one meter).

And in the example above, for both the observer on the ground and train, the length of moving ruler is elongated $\gamma$ times, so when use this ruler to measure dimension, the result Ld is:

$$
L d=L O / Y
$$

We examine the change of the standard ruler in the direction of movement. Obviously, the process can be reconstructed in the above-mentioned method. It is divided into 3 key frames, where T0 is the moment when the light is emitted, T1 is the moment when the light reaches the mirror, and T2 when the light reflects to the light source. At the moment of the source, the light is set to a single photon for the convenience of analysis. As shown in Figure 9. 
1

2

3

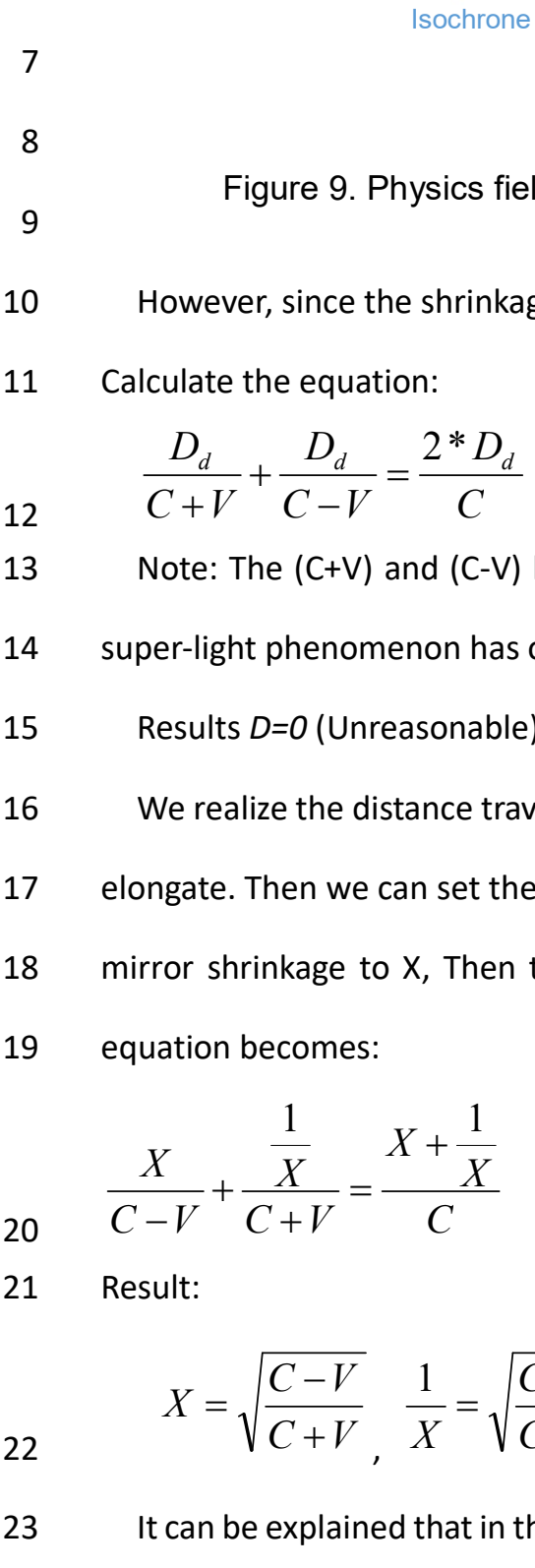

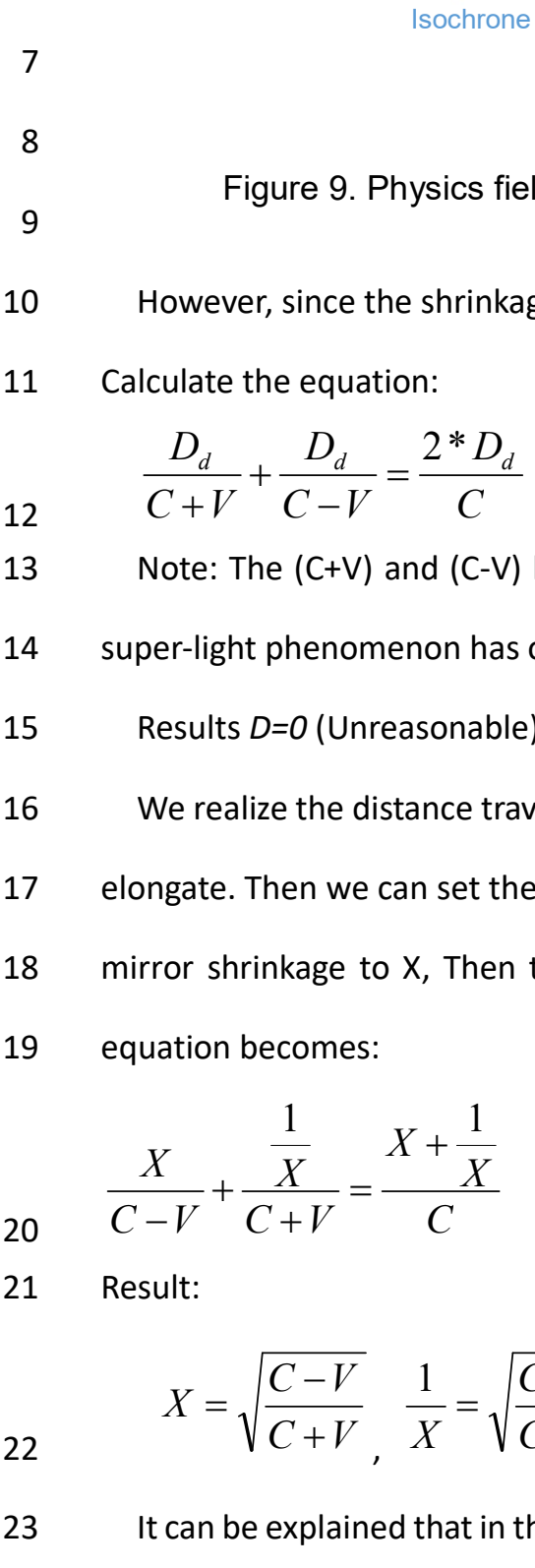

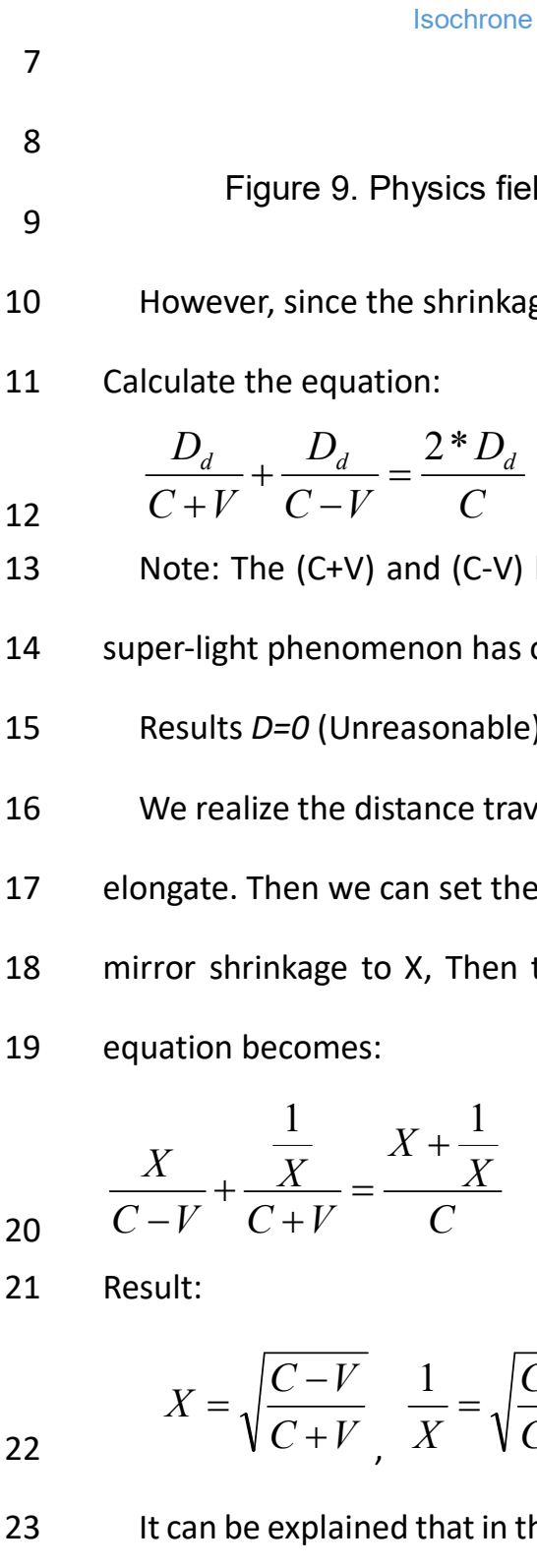

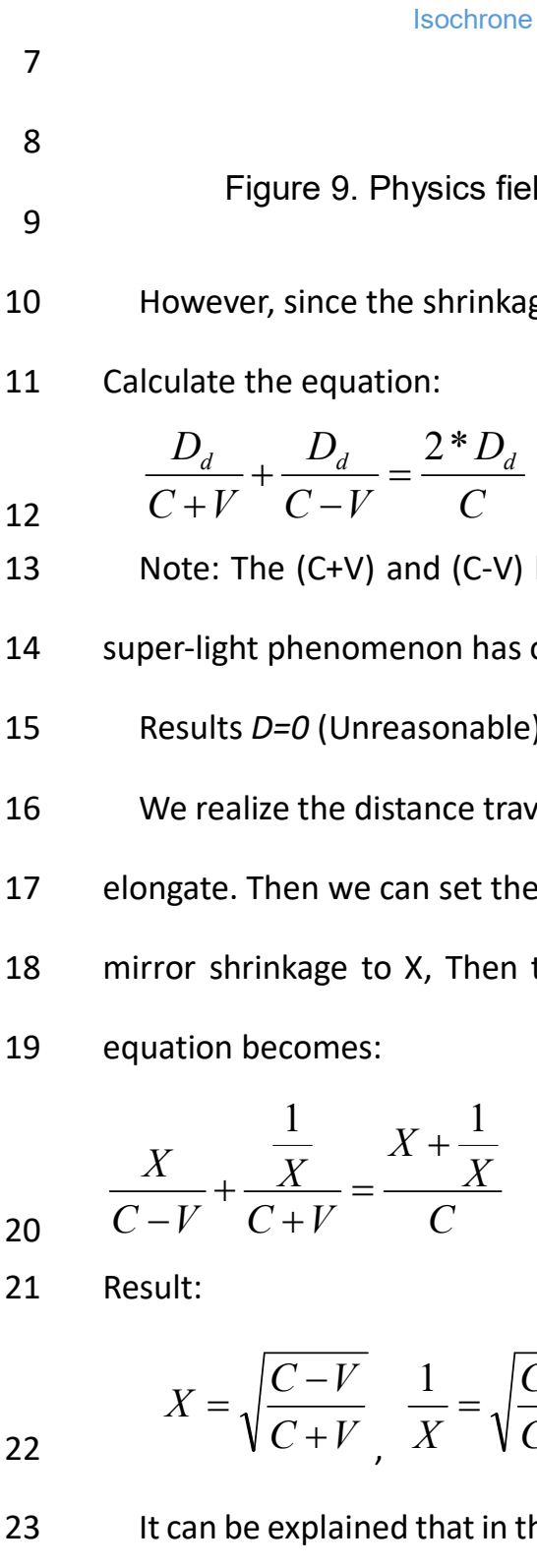

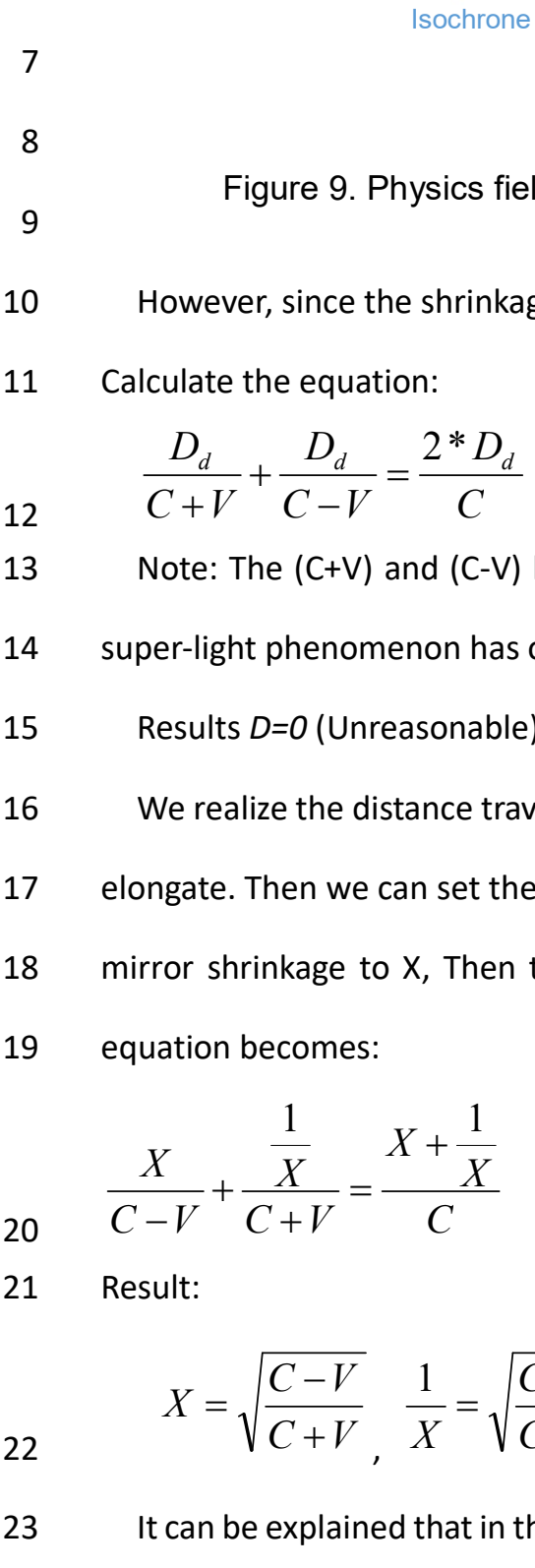

Figure
However, since
Calculate the equat
$\frac{D_{d}}{C+V}+\frac{D}{C}$
super-light phe
Results $D=0$
We realize the
elongate. Then
mirror shrinkage
equation becomes
$\frac{X}{C-V}+\frac{C}{C+V}$
Result:

24

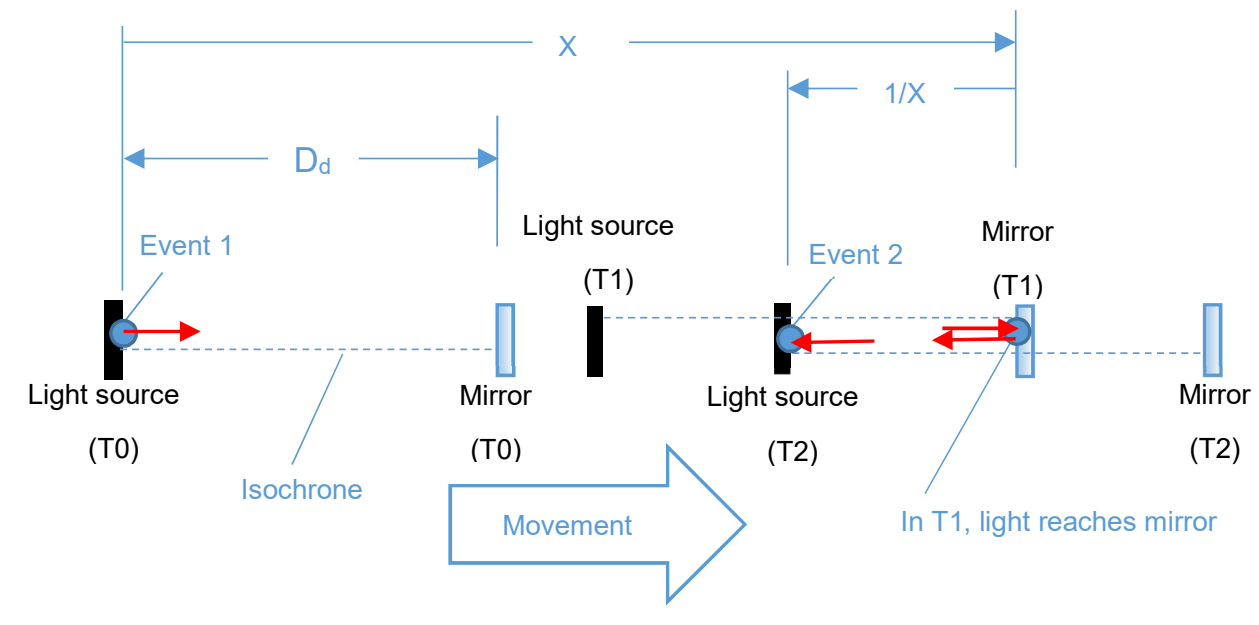

(T2) 
changes, the measured distance changes are as follows:

As far as the propagation of light in single direction, the length value elongation same as the direction of light movement is:

$$
L_{C}^{+}=L_{0} * \sqrt{\frac{C+V_{0}}{C-V_{0}}}
$$

The length contraction opposite to the direction of light movement is:

$$
L_{c}^{-}=L_{0} * \sqrt{\frac{C-V_{0}}{C+V_{0}}}
$$

Considering that the test distance generally requires a round trip of light (like a distance measurement by object), the average length change is:

$$
L_{d}=\frac{L_{c}^{+}+L_{\mathrm{c}}^{-}}{2}=\frac{L_{0}}{\gamma} \text {, the same as the other directions above formula (4). }
$$

Following Example in 8.4 will show the complexity in length change.

\section{Introduction of new concept of space-time}

Einstein's traditional Special Relativity explained that the time dilation in relative motion would introduce a lot of paradoxes and contradictions.

In view of the complexity of relative motion, it is difficult to correctly reconstruct the physical field within limited observations. Although the concept of ether should be discarded, in highvelocity motion, we can use the geometric center of the light emission as an absolutely rest frame of reference, which is easy for the analysis.

Based on this, we propose a new space-time concept here, including the following two basic assumptions:

- In the absolute rest frame of reference system, the velocity of light in all directions in the vacuum is $\mathrm{C}$, and this velocity is the limited velocity of the physical motion and information transmission.

- The physical laws observed in the correct physical field reconstruction of each frame of reference are all the same.

The following inferences:

A) The geometric center of light emission can be used as an absolute rest frame of reference.

B) In a correct physical field reconstruction, the time rate and spatial scale observed for the same 
device are the same no matter which frame of reference is used.

C) For the absolute rest frame of reference system, a device or object has:

TO: Called Rest Time.

LO: Called Rest Length.

After the device enters a state of uniform motion, no matter which frame of reference is observed, the device has:

Vo $<C$ : Called object's absolute Velocity reference to rest frame. Where $C=3 * 10^{8} \mathrm{~m} / \mathrm{s}$. Td: Called Dynamic Time, calculated by formula (3).

$L d$ : Called Average Dynamic length, calculated by formula (4).

Individually, in single direction, the length elongation same as the direction of light movement calculated by formula (5). The length contraction opposite to the direction of light movement calculated by formula (6).

D) Relative velocity

Unlike the Special Relativity, in new concept, for two different frame of references, the value of relative velocities is the vector summation generated by the absolute frame of reference respectively ( but each absolute velocity already limit by $C$ ), the following Example in 8.4 will show the complexity.

E) Momentum \& dynamic energy analysis:

Obviously, the traditional Special Relativity analysis is actually the absolute velocity rather than the relative velocity in momentum analysis, so most of the effects exist, but we change the $V$ in most formulas to the velocity $V O$ of the absolute frame of reference, and $T$ (time) in most formulas should also be confirmed to be whether the rest $(T O)$ or the dynamic $(T d)$.

In the Special Relativity, in order to avoid that the force $\mathrm{F}$ is kept on the object, which will produce stable acceleration and cause the velocity to exceed the $C$, and the concept of moving mass $M$ is in Special Relativity :

$M=M O * r$

In the new concept, the moving mass $M O$ (for the object as the frame of reference itself) does not change, but because $V O$ has been increasing, $T d$ will decrease to very small, so $V O=F{ }^{*} T d / M O$ is difficult to produce higher velocity, which is also limited by C. 
$12 \frac{C+U}{C}$

where $U<C$

$19 \frac{C+V}{C^{*} \gamma}$

$$
f^{\prime}=\frac{f}{\gamma} *\left(1+\frac{V}{C}\right)
$$

At low velocity, it is similar to the tradition, but at higher velocity, it is obviously different than

However, at this time, for the observer, the time $T d$ becomes $T O / V$, so the frequency $f$ change to: $f^{\prime}=f^{*} \gamma\left(1+\frac{U}{C}\right)$

When considering that the observer is rest, the light source moves at a velocity $\mathrm{V}$ (closer is positive, far away is negative). Considering $V$ is positive, the wave number increases, because the light source changes its time to $T D=T O / V$ causes less waves to be emitted, so the number of waves received by the observer during the rest frame of reference time TO becomes:

Special Relativity, and it needs the experimental verification.

\section{Explanations of the new space-time concept of common experiments \& calculation examples:}

8.1 The lifetime measurement of muons.

The average lifetime observed in lab when muons are rest is $2.2 \mu \mathrm{s}$. However, when the muons 
are moving at velocity $0.9994 \mathrm{C}$, the average lifetime measured is $63.51 \mu \mathrm{s}$. The new concept clearly points out that $2.2 \mu \mathrm{s}$ is close to the rest time. However, there may be another frame of reference $B$, the muon in B may have a shorter observation lifetime, indicating that the $B$ frame of reference tends to be absolutely rest than the earth.

\subsection{Hafele -Keating experiment}

The Hafele -Keating experiment [3]. They took four cesium-beam atomic clocks aboard commercial airliners. They flew twice around the world, first eastward, then westward, and compared the clocks against others that remained at the United States Naval Observatory. When reunited, the three sets of clocks were found to disagree with one another, their conclusion that their differences were consistent with the predictions of special and general relativity.

Actually in the part of special relativity it mentioned, it caused by the absolute motion, which can explained by the new concept is more acceptable.

8.3 Twin Paradox and other paradoxes.

The man who is in the absolute motion will be younger than who is in the planet clearly in new concept.

8.4 An example can reveal the difference between Galileo' concept or the Special Relativity and the new space-time concept in solve the problem:

Knowing that point $A$ is the absolute rest point, the observer walks for 1 year at a velocity of $V=0.6 C$ (Lorentz factor is 1.25 ) and shoots light forward while reaching point $B$. Set the light reaching point $C 1$ year later. Obviously, the distance between $A B$ can be calculated as 0.6 I.y. The distance between $B C$ is 1 l.y. The distance between $A C$ is 1.6 l.y.

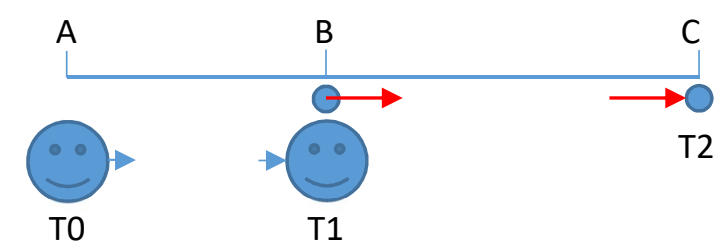

Figure 10. Physical field reconstruction of example

However, an equivalent problem has different solutions and answers:

Question 1:

Knowing that $A C$ is 1.6 I.y. apart, when the observer starts from $A$ at a velocity of $V=0.6 C, C$ emits light in the direction of $A$ at the same time. How long will the observer be able to observe the light? 


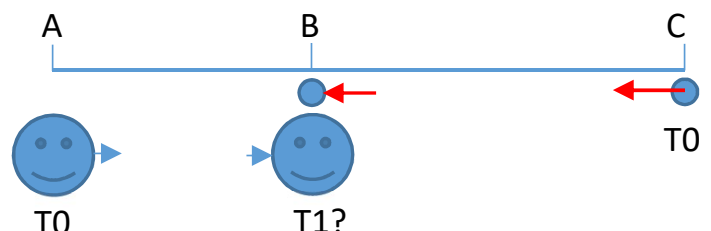

Figure 11. Physical field reconstruction of Question 1

Obviously, no matter what Galileo's concept or the correct Special Relativity or the new spacetime concept, the solution is:

$T=L /(C+V)=1.6$ I.y. $/(C+0.6 C)=1$ year

Note: The use of velocity $(C+V)$ here is only an arithmetic process, and does not mean that there is a super-light phenomenon in the physical field. Because there is no information transmitted from $\mathrm{C}$ to $\mathrm{A}$ at this time.

However, taking the observer as the frame of reference, the wrong opinion in the Special Relativity will think that the velocity cannot be summing. According the rules in Special Relativity, the velocity is also $(0.6+1) /(1+0.6)=C$, but at this time the Lorentz factor becomes infinite or even it is calculated at 1.25 , which makes it impossible to solve this problem correctly. In the new concept of space-time, there are two solutions to the motion system:

A) Assuming that the observer does not move, and the solution from the perspective of light is: Since the two moving directions are opposite, the total distance shrinks to:

$$
L_{C}=\frac{L_{0}}{\sqrt{\frac{(C+V)}{(C-V)}}}=\frac{1.6}{2}=0.8 l . y .
$$

Since the velocity of light does not change, the moving time $T d=T c=L c / C=0.8 \mathrm{l} . y . / C=0.8$ years. And the time of rest frame of reference is $T 0=1.25 * T d=1$ year. It will arrive after 1 year which is consistent with the preset.

B) Assuming that the light wave does not move, the solution from the observer is:

Since the two moving directions are opposite, the total distance is contracted by the Lorentz factor caused by V0 as $L d=L O / 1.25=1.6 / 1.25=1.28$ I.y.

The total velocity $V^{\prime}$ is $C+V=1.6 C$.

He believes that the required time is: $T d=L d / V^{\prime}=1.28 / 1.6=0.8$ year.

And the time of rest frame of reference is $T O=1.25 * T d=1$ year. 
5

6

7

8

9

10

11

12

13

14

15

\section{Summary}

The constant velocity of light and the way it spreads are characteristic of the cosmic context we live In view of the complexity of relative motion, it is difficult to correctly reconstruct the physical field within limited number of observations. In fact, almost all the motions discussed here are based on the absolute rest frame of reference, the physics field reconstruction can reflect the real physics phenomenon and also for analyzing the visual effects.

This new concept of space-time will refresh people's ideas about high-velocity moving objects and the results of observations of the universe.

\section{Reference}

2 [1] Fundamentals of Physics. Halliday, Resnick, Walker

[2] Einstein, Albert (1905). "On the Electrodynamics of Moving Bodies"

4 [3] Hafele, J. C.; Keating, R. E. (July 14, 1972). "Around-the-World Atomic Clocks: Predicted 5 Relativistic Time Gains". Science. 177 (4044): 166-168. 